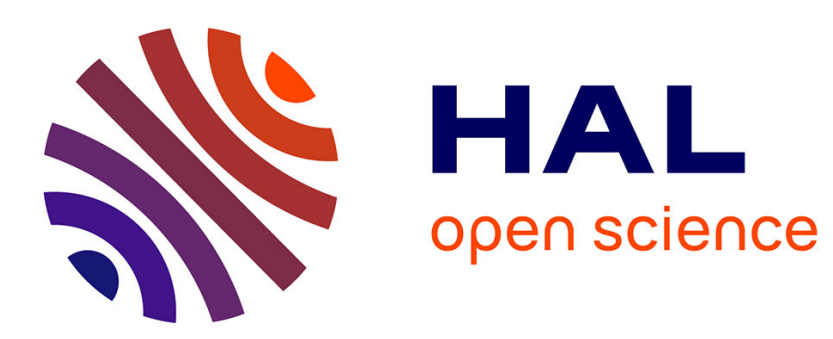

\title{
Le secteur privé égyptien et l'action publique: associations d'hommes d'affaires et démocratisation dans l'Égypte de l'infitah
}

Eric Gobe

\section{- To cite this version:}

Eric Gobe. Le secteur privé égyptien et l'action publique: associations d'hommes d'affaires et démocratisation dans l'Égypte de l'infitah. Cahiers d'études sur la Méditerranée orientale et le monde turco-iranien, 1999, 27, p. 213-225. halshs-00139538

\section{HAL Id: halshs-00139538 \\ https://shs.hal.science/halshs-00139538}

Submitted on 1 Apr 2007

HAL is a multi-disciplinary open access archive for the deposit and dissemination of scientific research documents, whether they are published or not. The documents may come from teaching and research institutions in France or abroad, or from public or private research centers.
L'archive ouverte pluridisciplinaire HAL, est destinée au dépôt et à la diffusion de documents scientifiques de niveau recherche, publiés ou non, émanant des établissements d'enseignement et de recherche français ou étrangers, des laboratoires publics ou privés. 
Référence complète de l'article

«Le secteur privé égyptien et l'action publique: associations d'hommes d'affaires et démocratisation dans l'Égypte de l'infitah », Cahiers d'études sur la Méditerranée orientale et le mondé turco-iranien, $\mathrm{N}^{\circ} 27$, janvier-juin 1999, p. 213-225

\section{Le secteur privé égyptien et l'action publique : associations d'hommes d'affaires et démocratisation dans l'Égypte de l'infitah}

Éric GOBE*

L'affirmation et la croissance du secteur privé en Egypte avec l'ouverture (infitah) de l'économie égyptienne en 1974 soulèvent la question des modalités de son organisation et de sa représentation auprès de l'Etat. Dès la fin des années 1970, les hommes d'affaires égyptiens les plus importants ont commencé à s'organiser en se regroupant dans des associations. Lors de la décennie 1980, cette propension à constituer des groupements s'est accentuée et les organisations visant à représenter les intérêts des chefs d'entreprises se sont multipliées. Aussi, le développement du secteur associatif patronal nous conduit-il à nous interroger sur la capacité du secteur privé égyptien à peser dans un rapport de forces avec la bureaucratie étatique.

En fait, on peut prétendre d'emblée que la crise de l'Etat distributeur ou redistributeur, qui a conduit les autorités égyptiennes à reconnaître une nouvelle légitimité au secteur privé, n'a pas permis l'émergence d'un groupe social capable d'avoir une action politique représentant et servant les « intérêts » du groupe dans un espace public, qui lui-même serait le symptôme de l'autonomie politique de la bourgeoisie égyptienne vis-à-vis de l'Etat.

Comme le rappelle Jean Leca, "l'idée qu'une structure de classe abstraite, du seul fait de son existence dans la société politique, pourrait déstabiliser des régimes autoritaires, est liée au paradigme d'une société bourgeoise où les relations économiques et sociales sont médiatisées par la citoyenneté et représentées par des relations politiques ${ }^{1}$.

De manière plus générale, c'est l'échec du «modèle stalino-rostovien de développement », pour reprendre l'expression de Michel Chatelus, qui a poussé les régimes arabes à se rallier de manière plus ou moins sélective à certains tenants du libéralisme économique. L'Egypte n'a pas échappé à cette pente.

Cependant, les gouvernants n'ont pas tenté de promouvoir des réformes de structures substantielles au travers d'une ouverture démocratique réelle. Ils ont, au contraire, développé des stratégies de survie qui sont destinées à répondre au niveau le plus bas possible, aux pressions de changements économiques et politiques, tout en maintenant l'hégémonie des élites gouvernantes ${ }^{2}$. Aussi le marché et le secteur privé ont-ils uniquement un caractère instrumental, leur réémergence n'étant pas accompagnée par le développement de l'Etat de droit.

C'est, bien évidemment, dans ce cadre qu'il convient d'aborder la question de la

\footnotetext{
${ }^{*}$ Chercheur à l'IREMAM/CNRS, Aix-en-Provence.

${ }^{1}$ Leca (J.), «La démocratisation dans le monde arabe : incertitude, vulnérabilité et légitimité », in Salame (Gh.) (dir), Démocraties sans démocrates. Politiques d'ouverture dans le monde arabe et islamique, Paris, Fayard, 1994, p 75.

${ }^{2}$ Brunberg (D.), «The Political Economy of Survival Strategies in the Arab World », Communication présentée à la conférence sur Political Liberalization and Democratization in the Arab World, Université de Montréal, 7-8 mai 1993.
} 
signification du développement des associations d'hommes d'affaires. La signification de l'existence des associations d'hommes d'affaires.

\section{La signification de l'existence des associations d'hommes d'affaires}

Les politiques publiques mises en œuvre par l'Etat égyptien ont eu pour objectif jusqu'à ce jour d'empêcher l'émergence de scènes de négociation ${ }^{3}$ autour desquelles les intérêts exprimés par les associations, de manière générale, auraient pu s'articuler.

La relation formelle entre les associations d'hommes d'affaires et le gouvernement est seulement de nature verticale, les propositions dans le domaine économique émanant exclusivement de l'Etat. Partant, les scènes de négociation, où des partenaires sociaux représentatifs d'intérêts négocient, sont inexistantes. En Egypte, les syndicats de salariés ont très peu d'autonomie et les relations entre ces derniers et les associations d'hommes d'affaires ne sont visibles que par leur absence. Depuis le début de l'ouverture économique on ne trouve la trace que d'une seule réunion entre les groupements représentant les hommes d'affaires et la Confédération des travailleurs égyptiens.

On pourrait, à la rigueur, discerner dans l'émergence d'associations d'hommes d'affaires les indices de la naissance d'une société civile (dans le sens de médiateur entre le centre politique et la société) en Egypte.

Plusieurs éléments viennent cependant contredire ce diagnostic : la jeunesse de ces groupements révèle la faiblesse de leur organisation. Ces associations sont encore, dans une large mesuré, l'expression des intérêts d'une minorité qui les considère comme leur propriété personnelle. Ce manque de légitimité des organisations d'hommes d'affaires a engendré une fragmentation des institutions censées représenter le secteur privé égyptien. Les leaders de l'Egyptian Businessmen's Association (EBA), la plus ancienne association d'hommes d'affaires, sont fortement liés à l'Etat et au secteur public. Les hommes d'affaires qui ont créé les associations, sont des personnalités entretenant des contacts avec l'élite gouvernante et qui, partant, n'ont pas besoin de s'organiser pour négocier avec le gouvernement ou l'appareil bureaucratique.

Les groupements organisés sont incapables de s'engager dans une confrontation directe avec l'Etat sur la question de leur autonomie ou sur 'd'autres sujets. Les porte-parole des associations d'hommes d'affaires considèrent le maintien de bonnes relations avec les autorités égyptiennes comme étant le meilleur moyen de recevoir dés faveurs du gouvernement dans le cadre de leur désignation au sein des organes consultatifs ${ }^{4}$.

Non seulement l'institutionnalisation des associations d'hommes d'affaires est faible mais encore elles revêtent principalement une dimension mondaine, les canaux d'accès au pouvoir bureaucratico-politique étant très personnalisés.

\section{La faiblesse des associations d'hommes d'affaires}

La genèse même des groupements d'hommes d'affaires nous renseigne sur leur faiblesse. Ce sont, avant tout, les liens d'amitié qui ont présidé à la création des associations d'hommes d'affaires : un petit noyau d'amis, ayant souvent étudié dans les mêmes universités ou travaillé ensemble dans le secteur public, a fondé son association.

Seule la Chambre de commerce américaine en Egypte (AMCHAM) échappe à ce

\footnotetext{
${ }^{3}$ Jobert (B.) et Muller (P.), L'Etat en action. Politiques publiques et corporatismes, Paris, PUF, 1987, pp. 159-170.

${ }^{4}$ Al-Sayyid (M. K.), «A Civil Society in Egypt », Middle East Journal, Vol. 47, n² 2, El 1993, p. 238.
} 
schéma en raison de sa très forte dimension interétatique. Elle cristallise principalement les intérêts des hommes d'affaires des deux pays.

En revanche, ce sont des réseaux informels qui ont été à l'origine de formation des autres groupements d'hommes d'affaires.

L'ancien premier ministre AAH, lui même propriétaire de plusieurs cabinets-conseils, affirme à ce propos : «C'est plus un groupe d'amis qu'un groupe professionnel, c'est un groupe d'amis qui pense qu'a Alexandrie ou au Caire, par exemple, ils sont très proches les uns des autres et donc font leur association $»^{5}$.

Les circonstances de la création de l'Egyptian Businessmen's Association nous éclairent sur la nature des liens qui unissaient entre eux les hommes d'affaires :

«Il y avait deux organisations sérieuses, semi-gouvernementales : la Fédération des industries égyptiennes et la Fédération des Chambres de commerce. Elles n'étaient pas très efficaces parce qu'en raison de leur statut elles étaient contrôlées à $90 \%$ par le ministère luimême. Dans la Fédération des industries aucune décision ne pouvait être prise sans l'accord final du ministère de l'Industrie, ce qui est un non-sens. C'est la même chose pour les Chambres de commerce, aucune décision ne pouvait être prise sans l'approbation du ministère de l'Approvisionnement. Le gouvernement a la majorité au conseil d'administration et a un impact sur le moindre problème. C'est pourquoi, lorsque le gouvernement a annoncé la politique d'ouverture économique au milieu des années soixante-dix, les gens travaillant dans le secteur public ont démissionné de leur poste et ont commencé à se mettre à leur compte. Vous pouvez comprendre qu'après plus de trente ans sans affaires privées [Cette affirmation est quelque peu exagérée : même pendant la période nassérienne, le secteur privé égyptien a continué à subsister.] ils n'étaient pas très puissants. Personnellement, ils étaient très forts, en tant qu'individus. Ils ont commencé à faire ce qui s'appelle encore l'Egyptian Businessmen's Association. Ils ont débuté au milieu des années 1970, dans une très petite organisation, en s'affirmant comme des personnages importants. Ils faisaient pour la plupart de l'importation ${ }^{6}$.

C'est dans ce cadre qu'a lieu l'enregistrement officiel de l'Egyptian Businessmen's Association auprès du ministère des Affaires sociales. Le comité fondateur se réunit, pour la première fois, au mois de mars 1977 sous la conduite de l'ancien ministre de l'Approvisionnement, Abdel Rahman Chazli. Cinquante personnes sont présentes lors de cette réunion du comité fondateur. Celui-ci pose les bases de l'organisation qui se fixe pour objectif général «de réaliser la politique de l'ouverture, de renforcer le rôle des hommes d'affaires et de participer avec son opinion, sa compétence, et ses efforts au renforcement de l'économie nationale ${ }^{7}$. Dès sa naissance l'association s'affirme comme différente des chambres de commerce (organismes semi-publics), donnant un sens particulier à l'enregistrement de ses statuts auprès du ministère des Affaires sociales.

En fait, les activités de l'association sont très faibles jusqu'en 1983, date de la parution de son premier rapport d'activités. Jusqu'en 1980, le nombre d adhérents se limite aux cinquante personnes du comité fondateur: L'association n'est pas encore structurée et ses membres ne paie généralement pas leur cotisation. Puis, après l'arrivée de Moubarak à la présidence de République et le vote de la loi 159 de 1981 sur les sociétés capitaux, le nombre

\footnotetext{
${ }^{5}$ Entretien avec AAH, octobre 1993.

${ }^{6}$ Entretien avec MG, l'un des membres fondateurs de l'EBA, mars 1994

${ }^{7}$ Déclaration du comité fondateur de l'EBA cité par 'Azmi (K.), «Jam'iyyat rijâl al-a'mâl misriyyin, al-namat al-jadîd min jamâ'ât al-masâlih fi al-mujtama’ al-misrî »[LAssociation des hommes d'affaires égyptiens : un nouveau type de groupes d'intérêts dans la société égyptienne], al-Fiker al-istrâtîii al-'arabî, Avril 1989, p. 168.
} 
des adhérents progresse pour atteindre 205 personnes $1983^{8}$. Cette même année, Saïd El Tawil, le président actuel de l'EBA, exige le paiement régulier de la cotisation. En 1984, l'association achète des bureaux modernes dans le bâtiment «la tour du Nil » (burj al-nîl). Elle dispose d'une l'autonomie financière et ne reçoit pas de subsides de l'Etat, cotisation étant passée de $500 £ \mathrm{E}$, en 1983, à $1000 \mathrm{£E}$ en 1985. La qualité membre est réservée aux Egyptiens, «propriétaires, directeurs généraux détenteurs ultimes de l'autorité ». Le candidat doit disposer d'une expérience d'au moins dix ans en tant que décideur. Il doit être parrainé par au moins deux membres de l'organisation et faire preuve de bonne moralité. Chaque membre du conseil dispose d'un droit de veto pour empêcher l'adhésion d'individu considéré comme indésirable. L'adhésion est individuelle et n'est pas incompatible avec un emploi dans le secteur public. De 205 en 1983, nombre d'adhérents est passé à 300 en 1986, à 385 en 1990, revenant, légère baisse à 372 en $1993^{9}$. Gamal El Nazer, le vice-président de l'EB explique cette stagnation du nombre des membres par la nécessité d'avoir d adhérents de qualité :

«Tout d'abord, l'EBA est très conservatrice à propos de ses membres, nous faisons très attention lorsque nous acceptons de nouveaux membres. Nous pouvons atteindre 1000 adhérents mais pas plus, nous insistons sur certains caractères qui limitent le nombre de membres. De plus, l'adhésion est personnelle, elle n'est pas pour les sociétés ${ }^{10}$.

Cette stagnation est en fait surtout le fruit d'une contestation de représentativité dont souffre l'EBA. Apparemment, l'EBA serait une organisation structurée capable, grâce à ses comités spécialisés et géographiques ${ }^{11}$, de jouer un rôle actif dans l'élaboration des politiques publiques comme l'affirme son comité fondateur. Mais ces comités sont en réalité des coquilles vides.

On retrouve de semblables structures organisationnelles au sein de l'Alexandria Businessmen's Association (ABA) qui a fait enregistrer ses statuts auprès du ministère des affaires sociales. Officiellement créée en 1988, elle est, en fait, l'émanation du comité des affaires rattaché à la Chambre de commerce d'Alexandrie. Né au sein de cette dernière en 1983, ce comité regroupait alors ceux qui se considéraient, dans les domaines de l'importexport, de la représentation commerciale, du tourisme et de la construction comme les hommes d'affaires les plus importants d'Alexandrie ${ }^{12}$. Il réunissait ainsi les chefs d'entreprise

\footnotetext{
8 Jam'iyyat rijâl al-a'mâl al-misriyyin, al-taqrir al-sanawî wa dalîl al-a'dâ' li-jam'iyyat rijal al-a'mâl al-misriyyin [Egyptian Businessmen 's Association, rapport annuel et annuaire des membres], Le Caire, Dâr al-'âlam al-'arabî, 1983, p. 6-31.

${ }^{9}$ Egyptian Businessmen's Association Members, 1993.

${ }^{10}$ Entretien avec Gamal El-Nazer, mars 1993.

11 L'EBA exerce son activité à travers des comités spécialisés et géographiques. Il existe environ onze comités sectoriels, couvrant les différents secteurs de l'économie (industrie, agriculture, construction, exportation, importation, investissement, tourisme, douane et fiscalité, transports et communications, financement et crédit, législation économique). Constitués en général de sept membres, ces comités sont censés préparer des études sur les différentes lois à caractère économique et de surveiller la manière dont elles sont appliquées. Le nombre de comités géographiques peut varier selon les années, mais il s'élève en général à huit (égypto-canadien, égypto-français, égyptoallemand, égypto-jordanien, égypto-italien, égypto-belge, égypto-hongrois). Ces derniers ont pour objectif de renforcer les relations d'affaires avec l'étranger. Les membres du comité rencontrent chaque année des délégations d'hommes d'affaires des organisations patronales des pays concernés en alternance, une année en Egypte, la suivante dans l'autre pays. L'assemblée générale se réunit une fois l'an. Le conseil d'administration y présente le rapport annuel et le compte budgétaire de l'année écoulée et l'on procède à l'élection d'un nouveau conseil d'administration. Ses membres sont élus à bulletin secret pour une durée de trois ans et doivent être renouvelés par tiers chaque année. 12 Qandil(A.), «Jamâ'ât al-masâlih wa al-intikhâbât : dirâsat hâla li-l-lajna al-iqtisâdiyya li-rijâl al-a'mal bi-lIskandariyya » [Les groupes d'intérêts et les élections : étude de cas du comité économique des hommes d'affaires d'Alexandrie], in Yassin (S.) et Dessouki (A. E. H.) (dir.), Intikhabât majlis al-sha'b, 1987: dirâsa wa tahlil [Les élections à l'Assemblée du Peuple, 1987], Le Caire, Centre d'études politiques et stratégiques d'al-Ahrâm, 1988, p. 62.
} 
du secteur privé qui voulaient se distinguer du commun des commerçants d'Alexandrie, obligatoirement membres de la Chambre de commerce.

Sous la houlette des hommes d'affaires Mohamed Ragab et de Ibrahim Kamel SidAhmed, le comité accède à l'indépendance par rapport à la Chambre de commerce ${ }^{13}$. Le ministère des Affaires sociales accepte d'enregistrer la nouvelle organisation patronale sur ses registres. L'association prend alors le nom d'Alexandria Businessmen's Association ${ }^{14}$.

La dernière organisation créée est le Club d'Affaires Franco-Egyptien (CAFE). Les modalités de sa naissance montrent encore l'importance de; réseaux d'anciens élèves. AEH, administratif du CAFE, indique, de manière indirecte, le rôle de socialisation des élites égyptiennes, dans la langue de Molière, qu'ont pu jouer les écoles de Jésuites, ainsi que les circonstances de la création du CAFE :

«Le directeur du poste commercial, le poste d'expansion économique au Caire, M Hédan, a pensé, à un certain moment, qu'il devait exister en Egypte une structure commerciale et économique parallèle aux structure étatiques ou officielles. En regardant autour de lui, il a vu que, du côté français, il y avait les conseillers du commerce extérieur qui étaient et même temps des dirigeants d'entreprises françaises et des expatriés français travaillant en Egypte. Du côté égyptien, il y avait des associations d'anciens élèves des écoles françaises jésuites qui ont pris contact avec les anciens élèves devenus hommes d'affaires. Ils ont vu ensemble l'opportunité de monter une association qui serait une sorte de Chambre de commerce franco-égyptienne. C'est une idée qui travaillait beaucoup de gens depuis longtemps $\gg$.

A la fin des années 1970, des villes nouvelles ont été créées pou désengorger le Caire, puis Alexandrie, de leur population et de leur industries. Tout un ensemble d'exemptions fiscales a été mis en place pour attirer les investisseurs industriels dans ces villes nouvelles. Celle de Dix de ramadan est la première à avoir été créée en 1979. L'association des investisseurs rattachée à cette ville a vu le jour en 1986 sous l'impulsion de l'industriel le plus important du pays, Mohamed Farid Khamis. Les trois autres villes nouvelles sont, par ordre chronologique : Six octobre, Sadat (1986) dont les associations ont été fondées toutes les deux en 1989, et Burj al-'Arab.

Les associations d'investisseurs ont moins d'adhérents que les autres organisations patronales, parce qu'elles représentent les intérêts des seuls industriels des villes nouvelles. Les droits d'entrée s'élèvent à $300 \mathrm{fE}$. Pour être membre, il faut posséder une entreprise installée dans la ville concernée. Les conseils d'administration ont quinze membres (sauf l'Association des investisseurs de Six octobre qui en a treize). Environ soixante industriels ont adhéré à l'Association des investisseurs de Dix de ramadan, mais ce sont les plus puissants.

Un examen des structures de ces groupements fait apparaître qu'ils disposent de peu de ressources organisationnelles. La forme même des organisations renseigne sur leur faible capacité à articuler les éventuelles demandes des adhérents. Ces associations n'ont pas suffisamment de ressources organisationnelles pour constituer des "groupes spécialisés dans le courtage d'intérêts ${ }^{16}$.

La politologue américaine Eva Bellin fait remarquer que les organisations regroupant les hommes d'affaires peuvent être une source de pouvoir dans la mesure où, dans les pays en voie de développement bien plus qu'ailleurs, les données sont rares et souvent incomplètes.

\footnotetext{
${ }^{13}$ Entretien avec HAW, membre du conseil d'administration de l'ABA, avril 1994.

${ }^{14}$ Elle a changé de nom en mars 1994 pour s'appeler Alexandrie Business' Association.

${ }^{15}$ Entretien avec AEH, avril 1 993. Par ailleurs, le CAFE annonce 420 membres et cotisation s'élève à 900 LE.

${ }^{16}$ Offerlé (M.), Sociologie des groupes d'intérêt, Paris, Montchrestien, 1994, p. 42.
} 
Une association d'hommes d'affaires, pourvue d'une équipe de chercheurs compétents, peut exercer une influence non négligeable en rassemblant toutes les informations statistiques et en les utilisant pour étayer sa vision de l'économie. Eva Bellin cite l'association des hommes d'affaires turcs, la TUSIAD, dont les études économiques très précises, bien documentées et largement diffusées, contribuent à former l'opinion des hauts-fonctionnaires et des hommes politiques turcs ${ }^{17}$. Ce n'est évidemment pas le cas des organisations patronales égyptiennes.

Le personnel de ces associations est peu nombreux, l'EBA et l'ABA disposent de cinq employés tandis que le CAFE en a quatre. L'organisation la mieux pourvue en personnel est 1'AMCHAM avec 16 employés. Cette faiblesse structurelle des associations marque encore bien plus les associations d'investisseurs, à l'exception de celle de Dix de ramadan. L'Association de la ville de Sadate, créée en 1989, a utilisé le bureau de son président comme lieu de réunion jusqu'en 1992, année où elle s'est enfin installée dans des locaux lui appartenant en propre dans la partie résidentielle de la nouvelle ville industrielle ${ }^{18}$. Dix de ramadan est le mieux structuré des groupements d'industriels et, sous l'impulsion de son président, ses membres ont formé une société d'exportation de leurs produits.

C'est incontestablement l'AMCHAM qui dispose du plus de ressources organisationnelles, mais elle a le défaut d'être une organisation binationale: "l'AMCHAM est bien mieux organisée que les autres associations. Elle a quand même le défaut d'être américanisée. Il manque alors la force de conviction d'une association d'hommes d'affaires $100 \%$ égyptienne $» 19$.

Cette faiblesse des ressources à la disposition des associations a un impact sur leur fonctionnement, sur les modalités de participation des membres et sur la répartition du pouvoir en leur sein.

\section{Participation et pouvoir dans les associations d'hommes d'affaires}

Les modalités de participation et d'organisation du pouvoir reflètent la prégnance de liens de type «primaire» au sein de ces associations d'hommes d'affaires. L'existence du groupement secondaire n'a pas encore fait apparaître un transfert d'allégeance, même si le monde associatif patronal évolue rapidement, depuis le milieu des années quatre-vingt, avec l'émergence d'un capitalisme industriel de plus en plus affirmé.

Les noyaux des associations sont, au moment de l'établissement de l'organisation, plutôt restreints (exception faite du CAFE, qui dès sa première année de fonctionnement, regroupait plus de 300 membres).

Il existe une dimension patriarcale dans le fonctionnement d'associations où le pouvoir est monopolisé par une oligarchie « d'anciens ».

Le renouvellement des membres des conseils d'administration est très faible: Les membres du conseil d'administration sont quasiment les mêmes depuis sa création.

Les dirigeants de l'Egyptian Businessmen's Association, dont nous connaissons l'âge, représentent les générations les plus anciennes de la bourgeoisie d'affaires égyptienne, puisqu'ils ont tous plus de 55 ans. Cette appropriation de l'association par la première génération d'hommes d'affaires de l'organisation est dénoncée par certains membres plus jeunes qui s'estiment bridés dans leurs initiatives. MKH, lui-même membre de l'EBA

\footnotetext{
${ }_{17}$ Bellin(E.), «Tunisian Industrialists and the State », in Zartman (I. W.) (dir.), Tunisia : The Political Economy of Reform, Boulder, Londres, Lynne Rienner Publisher, 1991, p. 58.

${ }^{18}$ Entretien avec SMS, membre éminent de l'Association des investisseurs de la ville de Sadate, mai 1994.

${ }^{19}$ Entretien avec HSE, ancien banquier, juin 1993.
} 
dénonce la dimension patriarcale de l'exercice du pouvoir dans cette organisation : "Seul je ne peux pas me battre contre ce groupe de vieux. C'est un peu un manque de démocratie aussi. Il y a un peu cet esprit patriarcal dans ces associations, le vieux, le grand, c'est celui qui est à la tête qui contrôle et qui est respecté ${ }^{20}$.

Les associations d'investisseurs étant plus récentes, il est difficile de tirer des conclusions. Si l'on considère la plus ancienne, celle de Dix de ramadan, on constate que le taux de renouvellement de ses dirigeants est très faible. On trouve un noyau dur d'individus quasi-immuable au conseil d'administration, autour de Mohamed Farid Khamis, inspirateur et président de l'association.

Au sein de l'EBA, l'unité est de rigueur lors de l'assemblée générale et dans les comités. Les rapports et mémorandums sont adoptés sans opposition. Le rapport annuel est systématiquement rédigé par Saïd El Tawil et n'est pas discuté. L'élection des membres du conseil d'administration se fait quasiment à l'unanimité :

L'adhésion à l'organisation donne surtout une visibilité sociale, notamment pour les hommes d'affaires de moindre envergure. Elle permet de se faire connaître quand on ne dispose pas d'un capital relationnel suffisant pour avoir accès, directement, aux hautes sphères de l'administration égyptienne.

Être membre de l'association, c'est avoir accès à des réseaux relationnels auxquels on ne peut pas prétendre autrement; mais dans le même temps, cela signifie la subordination à un individu ou à un groupe d'individus qui a un accès privilégié à l'élite politique.

Les anciens réseaux constitués pendant l'ère nassérienne n'ont pas disparu. Le passage d'une partie de la bourgeoisie d'Etat au sein du secteur privé n'a pas fait éclater les solidarités que constituent la $d u f a$ ' (c'est-à-dire les anciens élèves d'une même promotion ayant gardé des contacts et qui, sous Nasser, se rendaient des services pour se promouvoir les uns et les autres dans leur stratégie d'ascension sociale) et la shilla (elle consiste en un petit groupe dont les membres sont étroitement unis par des intérêts matériels communs, eux-mêmes parfois complétés par des liens affectifs) ${ }^{21}$.

On peut dire que l'association est victime du même syndrome néo-patrimonial que l'Etat, dans la mesure où il existe une appropriation et une personnalisation de l'association. Les organisations patronales s'identifient en la personne d'un homme d'affaires puissant dans son secteur ; c'est le cas de l'association des investisseurs de Dix de ramadan, qui est celle de Mohamed Farid Khamis, personne très proche du président Moubarak.

Ainsi, l'appropriation et la forte personnalisation des associations, couplées avec le désir des autorités égyptiennes de ne pas avoir qu'un seul interlocuteur, ont conduit à la multiplication du nombre des associations et à la contestation de la représentativité de l'EBA, dont les dirigeants sont accusés de collusion avec les autorités égyptiennes par les fondateurs des nouvelles associations.

\section{La signification de la multiplication des associations d'hommes d'affaires}

Le problème de la fragmentation et du manque de représentativité des associations d'hommes d'affaires est une des raisons de leur faiblesse. Aucune association n'a de véritable ancrage, ni de tradition, lui permettant de s'affirmer en tant que porte-parole légitime des hommes d'affaires.

\footnotetext{
${ }^{20}$ Entretien avec MKH, novembre 1993.

${ }^{21}$ Springborg (R.), The Ties that Bind : Political Association and Policy Making in Egypt, Ph. D., Startford

University, 1974, p. 59-62.
} 
L'appropriation des associations par leurs promoteurs, le clivage entre les générations d'hommes d'affaires, l'absence d'enracinement et de tradition dans l'organisation contribuent à cette fragmentation et à ce problème de représentativité des groupements d'hommes d'affaires.

Les adhésions passives ou bien la multiplication de celles-ci et la création d'autres associations, les membres centraux d'un groupement donné étant périphériques dans un autre, sont les symptômes de ce phénomène.

Nous pouvons également avancer l'hypothèse selon laquelle la multiplication des adhésions des hommes d'affaires dans les différentes organisations binationales est un moyen pour leurs membres de capter les ressources mises à leur disposition par le biais des protocoles financiers signés entre l'Egypte et l'Etat étranger concerné.

La différenciation entre les associations d'hommes d'affaires se fait également selon un clivage générationnel, bien que l'on ne possède pas de données suffisantes sur les membres du CAFE. Cette organisation est présentée souvent par la voix de son secrétaire ou de son président comme un nouveau type d'associations, en rupture avec les hommes d'affaires membres de l'EBA qui ont abandonné leurs fonctions de dirigeant de l'administration ou du secteur public pour créer leurs entreprises privées.

L'éloignement des centres de décisions bureaucratico-politiques serait une raison pour légitimer la constitution d'une nouvelle association. Les nouvelles générations d'hommes d'affaires, insuffisamment représentées dans les autres associations, créeraient ainsi la leur.

Cette fragmentation n'est pas seulement le fait des hommes d'affaires. Elle signifie également que l'Etat préfère gérer sa relation avec la bourgeoisie en reconnaissant plusieurs interlocuteurs afin d'éviter l'émergence d'un groupement trop puissant ${ }^{22}$.

\section{Conclusion}

Il convient de signaler que la faiblesse des associations d'hommes d'affaires n'est pas le propre de l'Egypte. Plusieurs auteurs qui se sont intéressés aux associations patronales dans d'autres pays arabes arrivent aux mêmes conclusions. Jean-Pierre Cassarino ${ }^{23}$ note, à propos de la Tunisie, que le progrès des réformes libérales peut difficilement être imputable à la mobilisation des «entrepreneurs » du pays. Les travaux de Saïd Tangeaoui sur le Maroc et ceux de Gregor B.M. Meiering concernant la Tunisie vont dans le même sens. Ce dernier fait remarquer que « les entrepreneurs sont plus habitués à faire le siège des administrations qu'a se comporter en groupe de pression $»^{24}$. Il précise que la principale organisation patronale tunisienne, l'UTICA, ne peut pas être considérée comme un lobby efficace. Cette dernière n'a pas, pour les entrepreneurs tunisiens eux-mêmes, la crédibilité d'un groupe de pression. Quant à Saïd Tangeaoui, il affirme que si «certains secteurs du grand patronat sont acteurs d'une

\footnotetext{
${ }^{22} \mathrm{Haw}$, membre du conseil d'administration de l'ABA, déclare à ce propos : «Je pense d'abord que l'Etat ne veut pas avoir une grande association. Vous savez que la création d'une association prend du temps. Je pense que, si les hommes d'affaires avaient présenté quelque chose d'uni au début, il y aurait eu une forte réticence du gouvernement à l'accepter. L'Etat, au début, ne voulait même pas de ces associations et pensait que la Fédération des industries et la Fédération des chambres de commerce suffisaient. Nous sommes au début et il est encore tôt pour parler de quelque chose d'unifié pour ces raisons ». Entretien avec Haw, membre du conseil d'administration de l'ABA, avril 1994.

${ }^{23}$ Cassarino (J. P.), « Garantir l'alliance entre la croissance économique et la stabilité sociale : Etat, entrepreneurs et "Mise à niveau" en Tunisie ", à paraître en 1999 dans le numéro des Cabiers de l'Orient consacré aux entrepreneurs arabes.

${ }^{24}$ Meiering (G.B.M), «Les entrepreneurs tunisiens », à paraître en 1999 dans le numéro des Cabiers de l’Orient consacré aux entrepreneurs arabes.
} 
dynamique associative », leur champ d'action reste malgré tout limité ${ }^{25}$.

Dans le cas particulier de l'Egypte, la relative autonomie accordée aux associations d'hommes d'affaires ne relève pas d'une forme de sociabilité apte à renforcer un tissu associatif articulant une sphère privée à un espace public, mais ressortit à un réaménagement partiel des relations entre l'élite politique et la bourgeoisie égyptienne.

En effet, si l'on se situe à un niveau macro-social, on constate que le processus de relative libéralisation, par lequel le régime de Moubarak accorde une marge d'autonomie à certains secteurs de la société égyptienne, concerne presque exclusivement les couches sociales supérieures. Ce processus n'est pas révélateur d'une libéralisation politique réelle, ni d'un renforcement global des organisations intermédiaires qui demeurent fragmentées en une série de poches ou d'îlots ${ }^{26}$.

Aussi, les éléments d'analyse que nous avons développés ici montrent que l'affirmation des associations hommes d'affaires égyptiens est largement insuffisante pour penser la démocratisation du régime égyptien. De manière générale, on peut dire qu'il ne suffit pas de faire référence aux relations entre la bourgeoisie et la bureaucratie pour appréhender une éventuelle transition démocratique. Il convient également de prendre en considération de manière plus large les rapports entre l'Etat et la société

Force est de constater que la revendication ouverte de démocratisation en Egypte n'est pas le fait des hommes d'affaires, mais plutôt d'intellectuels plus ou moins « islamistes » ou de « gauche » présents dans l'Organisation égyptienne des droits de l'homme ou le Syndicat des avocats. Même si la libéralisation économique implique une certaine décentralisation du pouvoir économique, elle ne crée pas nécessairement les acteurs sociaux qui seraient les vecteurs de la démocratisation: les hommes d'affaires égyptiens pourraient tout à fait s'opposer à la démocratisation si celle-ci donnait du pouvoir à des groupes considérés comme des adversaires virtuels de leurs «privilèges ».

\footnotetext{
25 Tangeaoui (S.), Les entrepreneurs marocains. Pouvoir, société et modernité, Paris, Karthala, 1993

${ }^{26}$ Dessouki (A. E. H.), «L'évolution politique de l'Egypte : pluralisme démocratique ou néo-autoritarisme », MaghrebMachrek, no 127, Janvier-Mars 1990, p. 14.
} 